\title{
Dual Power Management for Network Connectivity in Wireless Sensor Networks
}

\author{
Yanxia Rong ${ }^{1}$, Hongsik $\mathrm{Choi}^{2}$ and Hyeong-Ah Choi ${ }^{1}$ \\ ${ }^{1}$ Department of Computer Science \\ George Washington University \\ Washington DC \\ ${ }^{2}$ Department of Computer Science \\ Virginia Commonwealth University \\ Richmond, VA
}

email: yxrong@gwu.edu,hongsik@vcu.edu,hchoi@gwu.edu

\begin{abstract}
As the energy consumption in wireless sensor nodes is dominated by the radio transmission circuitry, the network configuration must be designed to minimize the power consumption by transmission radios. Sensor nodes are generally equipped with short-range radios that require low power consumption. But the current technology allows each node to adjust its transmission power. In this paper, we consider the dual power radios in which the radio of each node can be assigned high- or low-power during the network initializing stage. Our primary goal in such an assignment is to minimize the overall power consumption by radios to maximize the network lifetime while maintaining the full network connectivity, the most fundamental network functionality. Using a graph-theoretic approach, we formulate the problem as a "minimum subgraph" problem, shows its NP-completeness, establish upper and lower bounds on the optimum solution, and present a near-optimal heuristic algorithm and simulation results.
\end{abstract}

\section{Introduction}

Wireless sensor networks are ad hoc networks composed of a large number of small nodes. Such net- works are typically used for monitoring and surveillance functions. Once an "event" has occurred, the node needs to transmit some data collected from the event to a base station using a multi-hop path. Hence, one of the most fundamental functionalities of the network is to maintain its full connectivity. The sensor nodes in wireless sensor networks have a limited energy supply that usually cannot be renewed. Thus, the lifetime of a network is constrained by the amount of energy that is spent by the senor nodes in performing their operation of sensing, processing, and transmitting data to the control centers. Furthermore, the power consumption in sensor nodes is dominated by the radio transmission/reception circuitry. The power propagation models described in [10] imply that when the distance increases the power required to cover the distance increases dramatically. If there are two transmit power levels available for nodes to make the network connected, the higher power level used to connect the nodes far away is much larger than the lower power level that is used to connect the nodes in the neighborhood. Hence, the dual power management must be done to minimize the total number of nodes assigned high-power transmission. There has been much research related with our problem.

In traditional wireless protocol, the Distributed Control Function (DCF) defined in IEEE 802.11 [1], each node intends to use the same power level for 
transmission. Obviously, how to choose the common power level impacts the lifetime of the entire wireless networks. The common power level decision impacts the lifetime of the network but also the capacity of the network [2]. A type of problems arises here is called the power control problem, and the power control aims at choosing the transmit power to reduce the power consumption. In [3], a network layer protocol called COMPOW was proposed in which all the nodes eventually transmit at a common power level, which is lowest to connect the network. In [4] and [5], authors in addition focused on controlling the degrees of the nodes to save the power. Some researchers consider the power control in MAC layer as well. In [6], authors proposed a power controlled dual channel (PCDC) protocol which works on the MAC layer to allow each node to adjust its transmit power level. From another view of point, a clustering approach is proposed [7] where several transmit power levels are available at each node and the network is divided into several clusters.

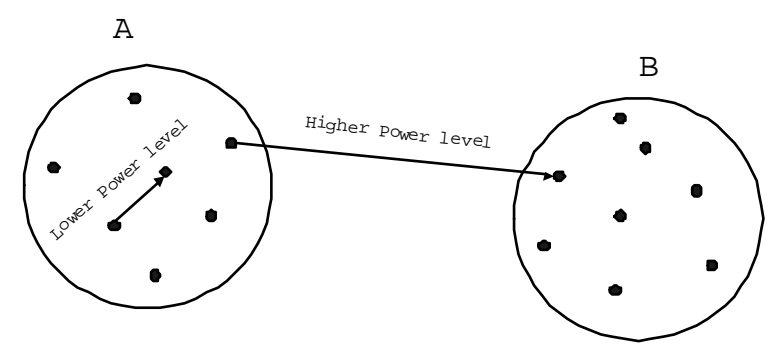

\section{Figure 1. Clusters with high- and low-power nodes}

In Figure 1, the nodes are grouped into two clusters $A$ and $B$. The nodes within each cluster are apparently closer to the nodes in the same cluster than those in different clusters. Thus, it is a waste of power to force all the nodes to transmit at the same power level that is at least able to cover the distance between the nodes in different clusters. Clustering is the idea that for those nodes close to each other, i.e., in a cluster, they can transmit at relatively lower power level and each cluster has at least one node that are capable of transmitting at higher power level to connect to different clusters. Thus, in Figure 1, a lower transmit power level is chosen for the communications within the cluster $A$ or $B$, and a higher transmit power level is used for the communications between the cluster $A$ and $B$. In [8], nodes are grouped into two categories: high-powered (HP) nodes and low-powered (LP) nodes, and the assignment is done to minimize the overall power while maintaining the full connectivity of the network. This is the same problem that we consider in this paper, but only simple heuristics are proposed in [8].

In this paper, we focus on a theoretical analysis of the problem. Using a graph-theoretic approach, our problem is formulated as a "minimum subgraph" problem. In Section 2, the network model considered in our problem is discussed, and a problem formulation is given. In Section 3, we show the NP-completeness of the problem, and lower and upper bounds of the optimal solution are given in Section 4. A near-optimal heuristic is presented in Section 5 followed by simulation results in Section 6. The paper concludes in Section 7.

\section{Network Model and Problem Formulation}

We assume that $N$ nodes are placed in a sensor field and the location of each node is given as a $x-y$ coordinates. We do not consider the mobility of nodes. Two power levels are given: $P_{H}$ and $P_{L}$. The transmission range using the high- and low-power radios are denoted by $r_{H}$ and $r_{L}$, respectively, where $r_{L}<r_{H}$. It is assumed that the network is fully connected if each node is assigned the high-power; hence, a feasible solution always exists.

Suppose each node $v$ has been assigned high- or low-power. Then, a directed graph $G=(V, E)$ is constructed as follows. The vertex set $V$ represents the $N$ sensor nodes and the edge set $E$ is defined such that a directed edge $(u, v) \in E$ if and only if the distance from $u$ to $v$ is no larger than $r_{L}$ (or $r_{H}$ ) if $u$ is assigned the low-power (or high-power). A directed graph $G$ is called strongly connected if and only if there exists a directed path from an arbitrary node to an arbitrary node in $G$. We refer a fully connected graph to a strongly connected graph, and these two terminologies are interchangeably used in this paper.

In order to minimize the overall power consumption in the network as much as possible, the overall transmit power used by all the $N$ nodes should be as little as possible. Therefore, our problem is to assign each 
node a power level $P_{H}$ or $P_{L}$ such that the summation of the assigned power to each node is minimum while maintaining the full connectivity of the network. We call this a Dual Power Management Problem (DPMP) which is formally defined as follows.

\section{Dual Problem Management Problem (DPMP)}

Instance: A set $\left\{\left(x_{i}, y_{i}\right) \mid 1 \leq i \leq N\right\}$ of the $x-y$ coordinates of the $N$ nodes; two transmit power levels $P_{H}$ and $P_{L} ; r_{H} \in R^{+}$represents the transmission range using $P_{H}$ and $r_{L} \in R^{+}$represents the transmission range using $P_{L}$.

Objective: To assign a power level to each node, $A: V \rightarrow\left\{P_{H}, P_{L}\right\}$, such that the corresponding directed graph $G=(V, E)$ is strongly connected, and $\sum_{v \in V} A(v)$ is minimized.

Before we consider any optimal solution to the DPMP, we first establish a condition for a feasible solution to exist. Define $\operatorname{dist}(i, j)(1 \leq i, j \leq N)$ to be the distance from node $i$ to node $j$. (Note that $\operatorname{dist}(i, j)=\operatorname{dist}(j, i)$.) Let $\operatorname{dist}\left(i_{0}, j_{0}\right)$ denote the smallest value such that a minimum spanning tree $T$ exists over the $N$ nodes where $\operatorname{dist}(a, b) \leq$ $\operatorname{dist}\left(i_{0}, j_{0}\right)$ for all $(a, b) \in E(T)$, where $E(T)$ denotes the edge set of $T$.

Lemma 1 A feasible solution to the DPMP exists if and only if $r_{H} \geq \operatorname{dist}\left(i_{0}, j_{0}\right)$.

Proof: Let $(u, v)$ be an edge in $E(T)$ such that $\operatorname{dist}(u, v)=\operatorname{dist}\left(i_{0}, j_{0}\right)$. By deleting $(u, v)$ from $T$, the $T$ is divided into two components, $A$ and $B$. From the well-known Kruskals' minimum spanning tree algorithm [9], it is noted that $\operatorname{dist}(i, j) \geq \operatorname{dist}(u, v)$ for any $i \in A$ and $j \in B$, i.e. edge $(u, v)$ is one of the minimum weighted edges between components $A$ and $B$.

Suppose there exists a solution to the DPMP and $r_{H}<\operatorname{dist}\left(i_{0}, j_{0}\right)$, i.e., the corresponding digraph $G$ is strongly connected. There then exist a directed edge $(a, b) \in E(G)$ connecting a node in component $A$ to a node in component $B$. This implies that $\operatorname{dist}(a, b) \leq r_{H}<\operatorname{dist}\left(i_{0}, j_{0}\right)=\operatorname{dist}(u, v)$, which contradicts that edge $(u, v)$ is one of the minimum weighted edges between components $A$ and $B$.

Conversely, assume that $r_{H} \geq \operatorname{dist}\left(i_{0}, j_{0}\right)$, and assign all the nodes high-power inducing a directed graph $G^{\prime}$. For all $(i, j) \in E(T)$, draw a directed edge from node $i$ to node $j$ and a directed edge from $j$ to $i$ so that we construct a directed graph $T^{\prime}$ on $T$. It is then noted that $T^{\prime}$ is strongly connected. Since $\operatorname{dist}(i, j) \leq \operatorname{dist}\left(i_{0}, j_{0}\right) \leq r_{H}$ for all $(i, j) \in E(T)$ and $T^{\prime}$ is a subgraph of $G^{\prime}, G^{\prime}$ must be strongly connected as well. Hence, assigning all the nodes high-power is a feasible solution to DPMP. This completes the proof.

\section{NP-Completeness of DPMP}

In this section, we show a polynomial time transformation from a known NP-complete problem, namely the vertex cover problem, to a decision version of the DPMP called the $D-D P M P$.

Vertex Cover Problem: A vertex cover of an undirected graph $G=(V, E)$ is a subset $S$ of $V$ such that for every edge $e \in E$, at least one end point of $e$ is contained in $S$. The goal of vertex cover problem is to find a vertex cover with the minimum size, i.e., the minimum number of nodes.

INSTANCE: Given a graph $G=(V, E)$ and an positive integer $k$.

QUESTION: Does $G$ have a vertex cover of size $\leq k$ ?

Given an arbitrary graph $G=(V, E)$ and an integer $k$, we will transform $G$ to an instance to the DPMP: a set $T$ of $x-y$ coordinates of $N$ nodes, $r_{H}$ and $r_{L}$, and an integer $c$, such that $G$ has a vertex cover of size $k$ if and only if there is a feasible solution to the DPMP with the number of high-power nodes being no more than $c$. (The actual value of $c$ will be discussed later.)

For each vertex $u$ in $G$, there is a corresponding component $C_{u}$ in $T$ called a vertex component that may include more than one node. For each edge $e$ in $G$, there is a corresponding component $C_{e}$ in $T$ called an edge component that may include more than one node as well. Any directed edge discussed below corresponds to the transmission using the high-power transmission.

There is a directed edge from component $C_{u}$ to component $C_{e}$ in $T$ if and only if the corresponding node $u \in G$ is incident with corresponding edge $e \in G$. In $T$, a vertex component may be connected to 
several edge components depending on the degree of of the corresponding node in $G$. If the vertex component is connected to several edge components, it represents that there is a common node inside the vertex component and this node can reach one node in each adjacent edge components using all high-power transmission. Initially, a directed bipartite graph for $T$ is constructed from $G$ ias shown in Figure 2.

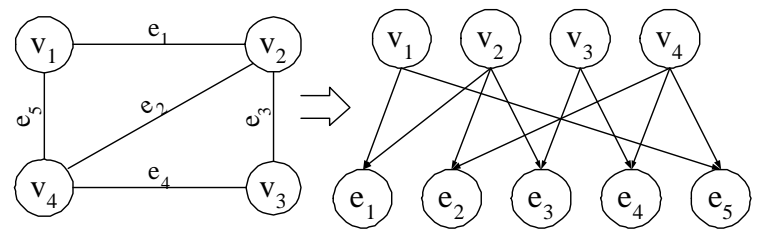

\section{Figure 2. Initial transformation from vertex cover problem to DPMP}

The directed edge from the a vertex component to an edge component means there is a node in the edge component falling into the high-power transmission range of a node in the node component. In addition to this initial construction, there is a component called top component which is shown in Figure 3 as $t$. Here, we assume that $t$ can reach one node in each vertex component using the high-power transmission. See Figure 3.

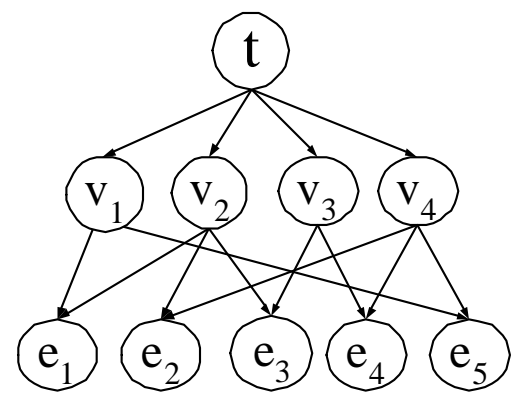

Figure 3. Transformation by adding a top component

Below the edge components, we add additional component each of which is one-to-one connected to each edge component. Below this level of components, we add another component called root component $r$. We assume that a node in each component can reach to a node in the root component. (Note that some of these nodes in the root components may be identical.) See Figure 4. Next, we add a chain of components from the root component to the top component. This chain of components are connected sequentially and not connected with either the edge components or the node components. This means that a node in each component of the chain can reach a node in its adjacent component downstream and a node in each component can be reached from a node in its adjacent component upstream. Lastly, we add one component called nearby component beside each vertex component, in which a directed edge from each vertex component to its corresponding nearby component exists and a directed edge from each nearby component to the top component also exists.

The Figure 4 shows the final $T$ after the transformation discussed above. It is recalled that all the directed edges correspond to the high-power transmission, and all nodes inside each components are connected through low-power transmission. It is easy to see that the procedure of transformation can be completed with polynomial time.

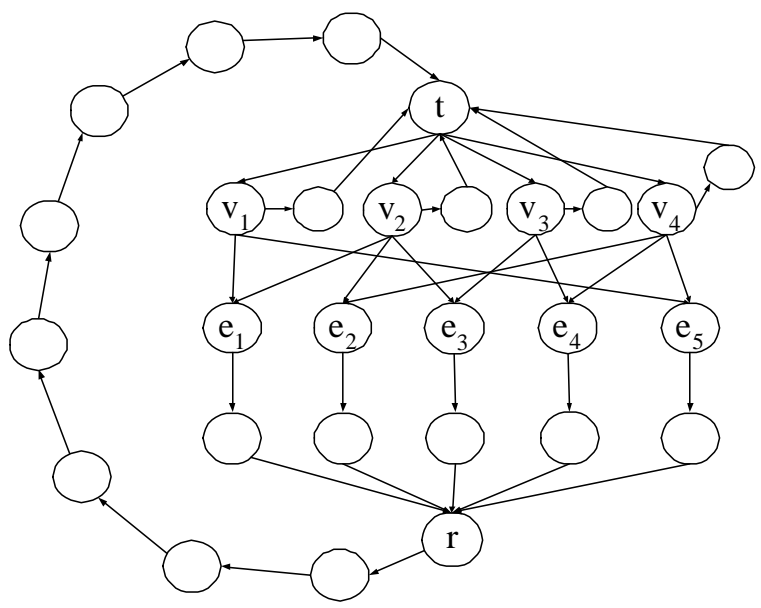

Figure 4. Final $T$ after transformation from vertex cover

We next proceed to prove the correctness of our transformation. Suppose there is a vertex cover $S$ in $G$ with $|S| \leq k$. We then assign high-power to the node in the top component so that it can reach all the vertex components. For each vertex $v_{i}$ in $S$ of $G$, we find its corresponding vertex in the edge component $C_{v_{i}} T$. 
By assigning this node high power that is connected to one or more edge components, we set up a communication path from the vertex component to its adjacent edge components. For instance, in the given case in Figure 2, a vertex cover set is $\left\{v_{2}, v_{4}\right\}$. In $T$, we assign high-power to nodes in component $C_{v_{2}}$ and $C_{v_{4}}$ such that all the edge components can be reached from one or more assigned high-power nodes. Note that except the vertex components and the top component, all the other components have only one outgoing edge, which means we have to assign one high-power node in each of these components to connect its downstream components. And for each vertex component, we have to assign one high-power node to reach their corresponding nearby components. Thus, for each vertex component corresponding to a node in vertex cover, we assign one node to connect the edge component and an additional high-power node to connect the nearby component. For the rest of vertex components whose corresponding vertices are not in the vertex cover $S$ of $G$, we only assign a high-power node to connect its corresponding nearby component. So the total number of high-power nodes is $N_{\text {high }}=$ number of components in $T+$ the size of $S$. (Note that the number of components in $T$ should be determined based on the layout of each nodes in a 2-dimensional space, and we do not give details here to keep the presentation simple.)

Conversely, suppose there exists a power assignment to nodes in $T$ with the $N_{\text {high }}=c$, where $c$ is defined as in the previous paragraph. Similarly to the above discussion, all the components except for the vertex components and the top component have only one outgoing edge; thus, only one high-power node in each component suffices to connect their downstream components. In the top component, the high-power has to be assigned to the node that reach all the vertex components. For each vertex component to reach its corresponding nearby components, the high-power has to be assigned to one node in each vertex component. So far, communication paths between the vertex components and the edge components have not been set up. To make the $T$ strongly-connected, each edge component must be connected to a vertex component. Thus the set of vertex components that include high-power nodes corresponds to a vertex cover set in $G$. The selected vertex components that are responsible for connecting the edge components have two assigned high-power nodes, one to connect the nearby component and one to connect the adjacent edge components. All other components have only one high-power node. Thus the corresponding vertex cover of $G$ has size $c$ - the number of components in $T$. This complete the proof.

\section{Upper and lower bounds on optimal solu- tion}

Since the DPMP is shown to be NP-complete, we proceed in this section to establish an upper and a lower bounds on the optimal solution.

Initially, we assign low-power to all of the $N$ nodes. We then observe that $u$ can erach $v$ using low-power if and only if $v$ can reach $u$ using low-power as well. Without loss of generality that the resulting directed graph is not strongly connected. Actually, the resulting graph has multiple components, and we let $K$ denote the number of components. The following results is then established.

Theorem 1 The optimal number of high-power nodes $O$ is such that $K \leq O \leq 2(K-1)$.

Proof: It is clear that each component must have at least one high-power node which establishes a lower bound, $O \geq K$.

To prove the upper bound, we construct a virtual undirected graph $G^{\prime}$ such that $G^{\prime}$ has $K$ vertices $C_{1}, \cdots, C_{K}$ and the edge set $E^{\prime}$ is defined as follows. There exist an edge $\left(C_{i}, C_{j}\right)$ if and only if there exists a pair of nodes $u \in C_{i}$ and $v \in C_{j}$ such that $u(v)$ can reach $v(u)$ using high-power transmission at $u$ $(v)$. We note that $G^{\prime}$ must be connected as a single component as otherwise, no feasible solution exists. Now, let $T^{\prime}$ be an arbitrary spanning tree of $G^{\prime}$, and we construct a set of high-power nodes as follows. For each edge $\left(C_{i}, C_{j}\right) \in T^{\prime}$, pick an arbitrary node $u \in C_{i}$ and an arbitrary node $v \in C_{j}$, and assign high-power to both $u$ and $v$. Repeat this process for each edge in $T^{\prime}$. Then, the resulting set of high-power nodes clearly provides a feasible solution to the DPMP, and the number of high-power nodes is at most $2(K-1)$. This establishes the desired upper bound 
on the optimal solution which completes the proof of the theorem.

For the optimal power assignment of the DPMP, the number of high-power nodes $N_{H}$ should be within the range that $K \leq N_{H} \leq 2(K-1)$. Therefore, we have

$$
\begin{aligned}
\sum_{v \in V} A\{v\} & =\left(N-N_{H}\right) \cdot P_{L}+N_{H} \cdot P_{H} \\
& =N \cdot P_{L}+N_{H} \cdot\left(P_{H}-P_{L}\right) \\
& \geq N \cdot P_{L}+K \cdot\left(P_{H}-P_{L}\right)
\end{aligned}
$$

and

$$
\begin{aligned}
\sum_{v \in V} A\{v\} & =\left(N-N_{H}\right) \cdot P_{L}+N_{H} \cdot P_{H} \\
& =N \cdot P_{L}+N_{H} \cdot\left(P_{H}-P_{L}\right) \\
& \leq N \cdot P_{L}+2(K-1) \cdot\left(P_{H}-P_{L}\right)
\end{aligned}
$$

Therefore, $N \cdot P_{L}+K \cdot\left(P_{H}-P_{L}\right)$ and $N \cdot P_{L}+$ $2(K-1) \cdot\left(P_{H}-P_{L}\right)$ are lower and upper bounds on the overall optimal power, where $K$ is the number of components as defined earlier.

\section{A near-optimal heuristic algorithm}

In the previous sections, we showed that the DPMP is NP-complete and established a lower and upper bounds on the optimal solution. In this section, we present a near-optimal heuristic algorithm. The performance of our heuristic algorithm is analyzed using simulation in the following section.

Our heuristic algorithm is based on the procedure that constructs the virtual graph discussed in Section 4. Initially, by restricting all the nodes to transmit at $P_{L}$, the $N$ nodes are grouped into several components and each component contains one or more nodes. We construct a virtual graph $G^{\prime}$ using these components. If any one node of a component $C_{i}$ can reach any node of component $C_{j}$ using the $P_{H}$, we draw an undirected virtual edge between component $C_{i}$ and $C_{j}$. From the assumption that if every node can transmit at $P_{H}$ the network is connected, we know that $G^{\prime}$ must be connected. Choose an arbitrary spanning tree of the virtual graph, denoted by $T^{\prime}$. Consider the leaf components of $T^{\prime}$ first, assign a high-powered node in the leaf component by assigning a high-powered node in its corresponding internal component in order to make the leaf component and the internal component can reach each other. When assigning the high-powered nodes in the internal components, we first consider nodes that have already been assigned high power previously. If we can find such assigned high-powered node in the internal component that can reach the leaf component being considered, we would prevent assigning an extra highpowered node. Delete the leaf component from the virtual graph, and repeat the above process. To make the number of high-powered nodes as few as possible, in each considering, those nodes that have already assigned $P_{H}$ always have higher priority than those not assigned $P_{H}$. Let $M$ denote the set of high-powered nodes. Initially, $M$ is empty. The detailed description of our algorithm is given below.

\section{A Heuristic algorithm for DPMP}

1: Divide the network into several components by restricting every node to transmit at $P_{L}$

2: Construct a virtual graph $G^{\prime}$ on the components

3: Choose a spanning tree $T^{\prime}$ of $G^{\prime}$ in terms of components

4: $M \leftarrow \phi$

5: Choose a leaf component $C_{u}$ of $T^{\prime}$ and its corresponding internal component $C_{v}$.

6: Consider all the pair of assigned high-powered nodes $i$ and $j$ in $C_{u}$ and $C_{v}$ respectively. If $i$ and $j$ can reach each other with $P_{H}$. Go to step 8 .

7: If a pair of nodes $i$ and $j$ in $C_{u}$ and $C_{v}$ respectively can can reach each other with $P_{H}$, assign this pair of nodes $P_{H} . M \leftarrow i, M \leftarrow j$.

8: Delete the component $C_{u}$ from the $T^{\prime}$

9: While $T^{\prime}$ has more than one components left, go back to step 5 .

Note that for each pair of leaf component and its corresponding internal component, we have assigned at most two high-powered nodes. Hence, our heuristic algorithm assigns at most $2 K$ high-powered nodes, where $K$ denote the number of components defined as before.

\section{Experimental Results}

We have implemented the heuristic algorithm presented in Section 5 and performed simulations on dif- 
ferent network sizes: $N=100,50,30,20$. The different networks that have different sizes are deployed on the same plane of size $1000 \times 1000$. Nodes in the network are deployed according to a spatial Poisson distribution on this plane. Given the deployment of the nodes, an interesting issue is how to select the $P_{H}$ and $P_{L}$, i.e., $r_{H}$ and $r_{L}$. Based on the lower bound shown in Lemma 1, we have calculated the minimum $P_{H}$ that can make the induced graph strongly-connected when every node transmit at $P_{H}$.

Given the network with $N$ nodes, we make a complete graph $G$. There is a weight associated with each edge, which represents the actual distance between the two nodes. We then construct a minimum spanning tree $T$ of $G$ using the Prim's algorithm. Thus, the $T$ has $N-1$ edges. Among these $N-1$ edges, we choose the maximum weighted edge. The minimum $P_{H}$ is the power whose corresponding transmission range is equal to the weight of this edge. It is not difficult to show that the $r_{H}$ calculated in this way is the minimum high power level to make the network fully connected. In each simulation, we calculate the minimum $r_{H}$ after the deployment of $N$ nodes. Given the value $r_{H}$, we vary the value of $r_{L}$ from $5 \%$ of $r_{H}$ to $80 \%$. Each simulation runs 50 times and the average value is obtained.

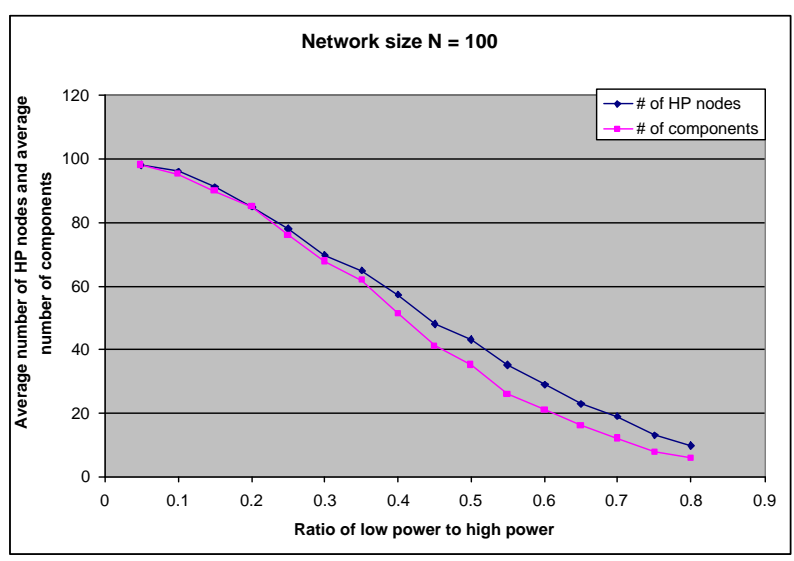

Figure 5. $N=100$

Figures 5, 6, 7, and 8, respectively, show the result when the network size is $100,50,30$, and 20. The simulation results for different network sizes depict a similar feature. When the $r_{L}$ is close to zero percentage of $r_{H}$, the number of components is close to the total number of nodes as most components contain

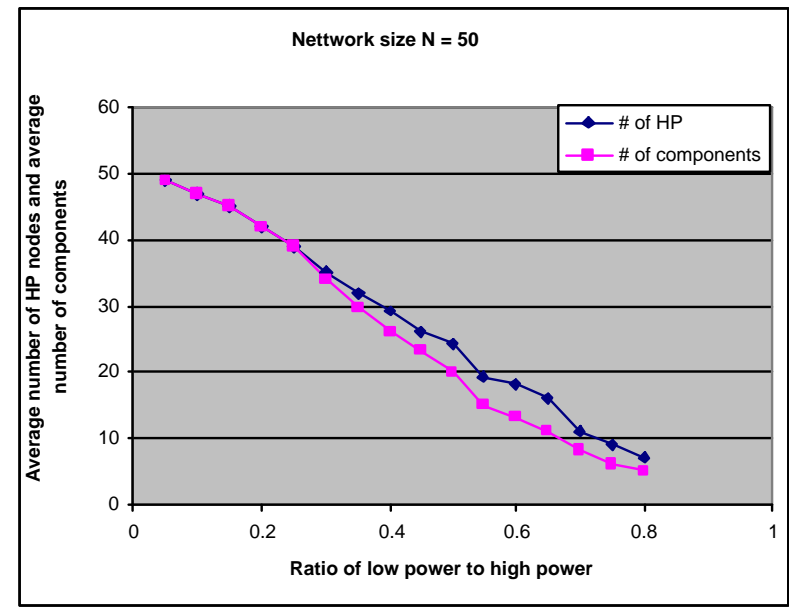

Figure 6. $N=50$

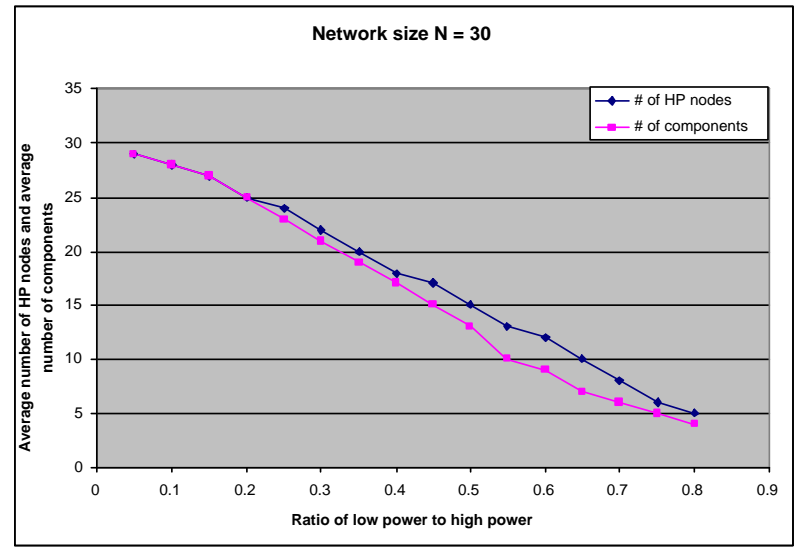

Figure 7. $N=30$

only one node; hence, the number of high-powered nodes is close to the total number of nodes. This result implies that if $r_{L}$ is too little compared to the $r_{H}$, each node can hardly reach any other node using lowpowers only. Consequently, it requires almost all the nodes to transmit at the high-power level. To the contrary, if $r_{L}$ is close to the $r_{H}$, for example $80 \%$ of $r_{H}$, the number of components is close to one, hence the number of high-powered nodes is close to one as well. However, if the gap between the $r_{H}$ and $r_{L}$ is not large enough, we spend too much power on $P_{L}$ and we lost the motivation to use dual transmit power levels.

We have also considered a specific application scenario in which the sensor field is divided into equal sized regions, and each local region has at least one sensor node. In our simulation, we divided the origi- 


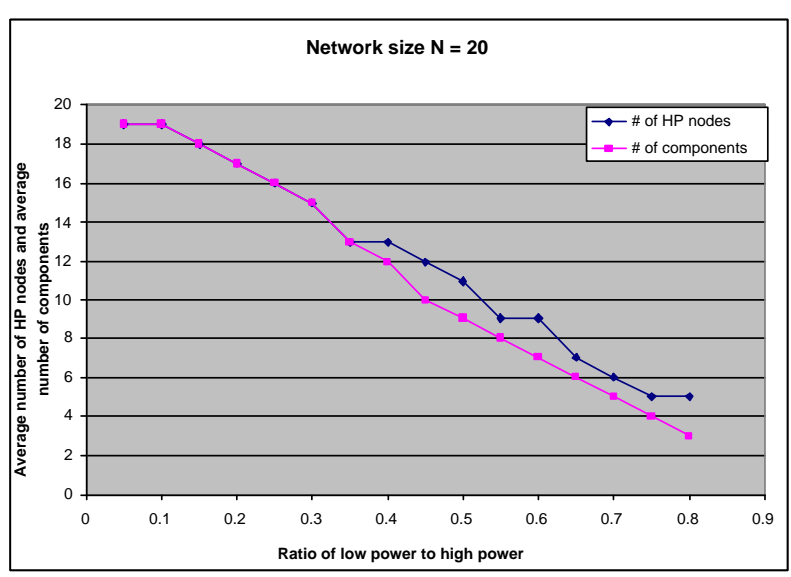

Figure 8. $N=20$

nal plane into 100 small regions and each region has at least 2 where 300 nodes in total are placed in the sensor field. Figure 9 shows results of this case. No significant difference is observed implying that a random, yet simpler, deployment would perform as well.

Note that the number of components shown in each figure is a lower bound on the optimal solution as discussed in Theorem 1. The result is shown in the Fig-

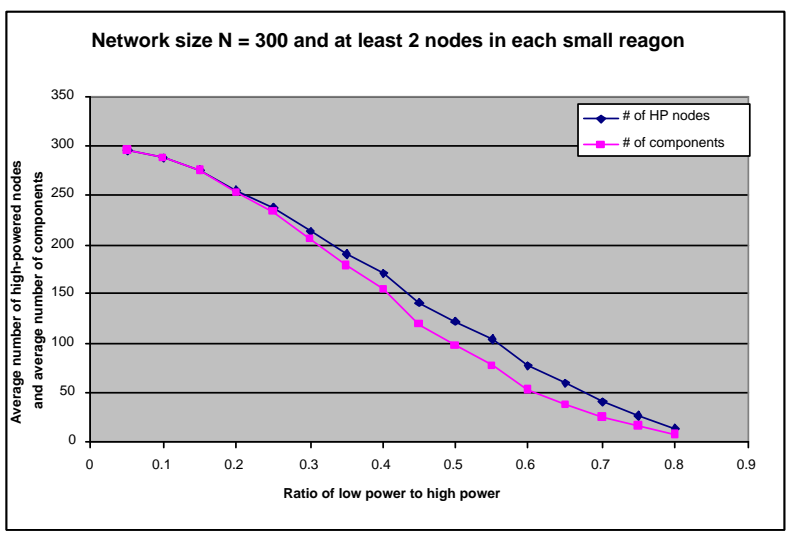

Figure 9. The plane is divided into 100 small regions and each region has at least 2 nodes

ure 9. This specific scenario didn't present big difference with the above scenarios, except that this kind of scenario intends to deploy the nodes more homogeneously.

\section{Conclusion}

In order to reduce the power consumption in wireless sensor networks, we would like to group the nodes into several clusters (components). In each cluster, the node can communicate with each other using relatively lower power transmission level. To guarantee the communications between different clusters, each cluster has to have at least one node that transmit at relatively higher power level. Given the two levels of transmit power, we have considered the problem of determining high-powered nodes and low-powered nodes so that the number of high-powered nodes is minimized while maintaining the full connectivity of the induced directed graph. We called the problem a DPMP.

We showed the NP-completeness of the DPMP, established a lower and an upper bound of the optimal solution to the DPMP, developed a near-optimal heuristic algorithm, and presented simulation results to analyze the performance of our heuristic algorithm.

For the future work, we plan to develop a distributed version of our heuristic algorithm. Another interesting research direction is to consider network reconfiguration, i.e., reallocate high- and low-power nodes after a certain period of time to maximize the network lifetime. It would be also interesting to investigate the feasibility and complexity when more than two power levels are introduced, in particular, when the power management is dynamically done.

\section{Acknowledgement}

This work was supported in part by U.S. Army Research Office under contract DAAD 19-02-D-001, TCN02-007.

\section{References}

[1] International Standard ISO/IEC 8802-11; ANSI/IEEE Std 802.11, 1999 Edn. Part 11: wireless LAN Medium Access Control (MAC) and Physical Layer (PHY) specifications.

[2] P. Gupta and P.R. Kumar, "The capacity of the wireless networks", IEEE Transactions on Information Theory, vol. IT-46, 2000.

[3] S. Narayannswamy, V. Kawadia, R. S. Sreenivas, and P. R. Kumar, "Power control in ad-hoc net- 
works: Theory, architecture, algorithm and implementation of the COMPOW protocol", European Wireless Conference, 2002.

[4] R. Ramanathan and R. Rosales-Hain, ”Topology control of multihop wireless networks using transmit power adjustment", Proceedings of INFOCOM, 2000.

[5] T. A. ElBatt, S. V. Krishnamurthy, D. Connors, and S. Dao, "Power management for throughput enhancement in wireless network ad-hoc networks", IEEE International Conference on Communications, 2000.

[6] A. Muqattash and M. Krunz, "Power controlled dual channel (PCDC) medium access protocol for wireless ad hoc networks", INFOCOM 2003.

[7] T. J.Kwon and M.Gerla. "Clustering with power control", Proceedings of the IEEE Milicom conference, volume 2, 1999.

[8] P. Sholander, G. Frank and A. Yankopolus, "Energy-efficient network techniques for wireless sensor networks", Milicom 2003.

[9] E. Horowitz, S. Sahni and S.Rajasekaran, "Computer Algorithms", W. H. Freeman and Company, 1998.

[10] T. Rappaport, "Wireless Communications: Principles and Practice", Prentice Hall, 1996. 